Originalveröffentlichung in: Bernd U. Schipper (Hrsg.), Ägyptologie als Wissenschaft. Adolf Erman (1854-937) in seiner Zeit, Berlin 2006, S. 258-275

\title{
Adolf Erman und die internationale Ägyptologie
}

\author{
Louise Gestermann
}

Eine detaillierte Aufarbeitung des Nachlasses von Adolf Erman in der Staatsund Universitätsbibliothek (SuUB) Bremen steht noch aus, und so greift auch der folgende Beitrag nur einen von vielen möglichen und ganz unterschiedlich gelagerten Gesichtspunkten auf, unter denen sich der Nachlass betrachten ließe. Unter dem Stichwort „Adolf Erman und die internationale Ägyptologie“ soll zweierlei zur Sprache kommen, wobei zugleich gesagt werden muss, dass sich beides nicht klar voneinander trennen lässt. Es wird zum einen um die Kontakte gehen, die Erman zu Fachkollegen im Ausland hatte, um die Korrespondenzpartner selbst sowie um die Art dieser Kontakte und die Inhalte, über die sich Kollegen in ihren Briefen mit ihm austauschten (1). Zum zweiten sollen unter Hinzuziehung von Informationen aus dem Nachlass Einschätzungen dazu vorgelegt werden, wie sich das Verhältnis der deutschen zur internationalen Ägyptologie zu Zeiten Ermans gestaltete (2).

Die folgenden Ausführungen können diese beiden Themen bzw. Gesichtspunkte nur schlaglichtartig beleuchten. Zum einen müssen wir davon ausgehen, dass die Auswertung des Nachlasses Einblicke gewähren wird, die sich zurzeit nicht oder nicht deutlich genug abzeichnen. Zum anderen haben wir uns zu vergegenwärtigen, dass wir in der Regel nur die Briefe des Gegenübers, des Korrespondenzpartners, besitzen, Erman schweigt in seinem Nachlass weitgehend. Es gibt nur wenige Antwortschreiben oder Entwürfe dazu, die dann offizielle Vorgänge betreffen. ${ }^{1}$ Vorauszuschicken ist des Weiteren, dass der Nachlass möglicherweise nicht ganz vollständig ist. ${ }^{2}$

Mitunter finden sich auf den Schreiben, die Erman erhalten hat, kurze Vermerke, mit denen Erman Stichworte seiner Antwort fest hielt.

2 Diesbezügliche Überlegungen sind bereits vorgetragen worden, wobei insbesondere das Fehlen etwaiger Briefe von Hermann Junker und Hermann Kees sowie die fehlenden Kondolenzschreiben an die Familie genannt wurden, GESTERMANN \& SCHIPPER 2004, 40, 41 mit Anm. 19. Die internationale Korrespondenz zu beurteilen ist schwierig, mitunter fallen Kleinigkeiten auf. So berichtet ERMAN 1929, 244, davon, brieflichen Kontakt mit Urbain Bouriant gehalten zu haben. Die Angaben dazu sind eher vage, aber im Nachlass werden nur drei Briefe von Bouriant aus den Jahren 1887/1888 


\section{Die internationale ägyptologische Korrespondenz Adolf Ermans}

\subsection{Die Korrespondenzpartner}

In seiner Biographie aus dem Jahr 1929 äußert sich Adolf Erman zu seiner international zusammengesetzten Studentenschaft und schreibt in diesem $\mathrm{Zu}$ sammenhang: ${ }^{3}$

„Zu den deutschen Studenten kamen dann ausländische aus aller Herren Länder, Amerikaner wie Breasted, Reisner, Gillet und Caroline Ransom, Engländer wie Crum, Gardiner und Boylan, Niederländer wie Boeser, Russen wie Turajeff und Struve, Belgier wie Capart, Franzosen, Schweizer, Skandinavier und auch moderne Ägypter fanden sich zuweilen ein. Vielfach waren diese Ausländer reifere Männer, die ihre anderen Studien zu Hause abgeschlossen hatten und die nun nach Berlin kamen, um noch Ägyptisch zu lernen. Auch diese vereinigte das gleiche Streben mit uns und den anderen Studenten zu wirklicher Freundschaft, und bei allen hat diese auch die schwerste Prüfung in der Kriegs- und Nachkriegszeit überstanden."

Die genannten Studenten, die aus dem Ausland nach Berlin und zu Erman kamen, finden sich dann auch unter seinen Korrespondenzpartnern, wobei einige von ihnen zu denjenigen gehören, mit denen Erman einen langjährigen und umfangreichen brieflichen Kontakt hatte, so Walter E. Crum, Alan H. Gardiner, James H. Breasted, Caroline Louise Ransom Williams, Pieter Boeser und Jean Capart (s. Abb.). Auch Hans O. Lange, Eugène Dévaud und Vladimir Samionovich Golenischeff, die Erman in dem zitierten Abschnitt aus seiner Biographie nicht namentlich erwähnt, die aber zu wichtigen Korrespondenzpartnern wurden, waren Studenten bei ihm. Bei den anderen Kollegen hat sich der Kontakt auf unterschiedlichen, in der Korrespondenz häufig nicht ausdrücklich erwähnten und mitunter nur sehr schwierig zu rekonstruierenden Wegen ergeben.

aufbewahrt. Ein ibd. erwähnter Brief vom Sohn Bouriants findet sich im Nachlass gar nicht.

3 ERMAN 1929, 283. 


$\begin{array}{lll}\text { Nation } & \text { Briefe Karten } & \begin{array}{l}\text { Antwort } \\ \text { Erman }\end{array}\end{array}$

Walter Ewing Crum

$\begin{array}{lllll}1865-1944 & \text { GB } & 322 & 5 & 5\end{array}$

Hans Ostenfeldt Lange

$\begin{array}{llll}1863-1943 & \text { DK } & 203 & 3\end{array}$

Allen Henderson Gardiner

$\begin{array}{llll}1879-1963 & \text { GB } & 87 & 4\end{array}$

Francis Llewellyn Griffith

$\begin{array}{lll}1862-1934 & \text { GB } & 82\end{array}$

James Henry Breasted

$\begin{array}{llll}1865-1935 & \text { USA } & 80 & 12\end{array}$

Eugène Victor Dévaud

$\begin{array}{llll}1878-1929 & \mathrm{CH} & 62 & 16\end{array}$

Caroline Louise Ransom

$\begin{array}{lllll}\text { Williams } 1872-1952 & \text { USA } & 56 & 10 & 3\end{array}$

Édouard Henri Naville

1844-1926

$\mathrm{CH}$

Pieter Adriaan Aart Boeser

$\begin{array}{llll}1858-1935 & \text { NL } & 49 & 3\end{array}$

Eugène Charles Revillout

1843-1913

Gaston Camille Charles Maspero

$$
\text { 1846-1916 F }
$$

2

2

Jean Capart

$$
\text { 1877-1947 }
$$

B

44

6

Norman de Garis Davies

$\begin{array}{llll}1865-1941 & \text { GB } & 26 & 3\end{array}$

Vladimir Samionovich
Golenischeff 1856-1947
R
25
3
2

Abb.: Die internationalen ägyptologischen Korrespondenzpartner Adolf Ermans (Nachlass Ermans in der SuUB Bremen), vgl. dazu die Angaben bei KLOFT et al. 1982, 5894.

Auffälligerweise finden auch von den Ägyptologen aus dem Ausland, mit denen Erman eine umfangreiche Korrespondenz führte bzw. die zahlreiche Briefe an ihn richteten, nur wenige in seiner Biographie Erwähnung. Selbst zu den Verfassern der größten Briefkonvolute verliert er - abgesehen von dem zuvor zitierten Passus - kaum mehr als ein paar Worte. Lange, Gardiner und 
Breasted werden kurz in Zusammenhang mit dem Wörterbuch genannt (s.a. im Folgenden), Lange außerdem in Verbindung mit seiner Neubearbeitung des Weisheitsbuchs des Amenemope. ${ }^{4}$

Bisweilen offenbart sich bei einem Vergleich zwischen der Biographie Ermans und den Briefen, die ihm geschrieben werden, auch ein Missverhältnis zwischen dem brieflichen Kontakt, Umfang wie Inhalt der Briefe sowie der Wertschätzung oder besser Einschätzung des Kollegen, die man Erman auf Grund der Äußerungen in seiner Biographie unterstellen darf.

Mit Édouard Naville, dem Schweizer Ägyptologen, der schon bei Karl Richard Lepsius studiert hatte und eine Schweizer Ägyptologie begründete, lag Erman zeitlebends in einem Streit wegen des gegenseitig angenommenen Unverständnisses für die ägyptische Sprache. In seiner Biographie benennt Erman diesen Zwist recht offen und scheute sich auch nicht davor, Naville den „Dilettanten“ zuzurechnen, „die in England und anderswo mit Hieroglyphen spielten". 5 In den Briefen Navilles schlägt sich die beiderseitige Ablehnung indes nicht in dem Maße nieder, im Gegenteil sah sich Erman verschiedentlich veranlasst zu vermitteln, so zwischen Naville und Kurt Sethe. ${ }^{6}$

Gaston Maspero, der Direktor des Service des Antiquités in Kairo, war für die Arbeit der deutschen Ägyptologen in Ägypten immens wichtig. Erman selbst erfährt von ihm Unterstützung bei der Ausfuhr seiner Ankäufe für das Berliner Museum, die er 1885 bei seinem ersten Besuch in Ägypten getätigt hatte. ${ }^{7}$ Nicht nur in Worten, sondern auch in Taten wird Maspero von Erman gewürdigt: Auf Veranlassung von Erman wird Maspero zum Korrespondierenden Mitglied der Akademie. Die Briefe Masperos an Erman sind freundschaftlich, entgegenkommend und ohne Vorbehalte. Es ist vor allem Anerkennung und Wertschätzung für Erman, die sich in ihnen offenbaren. Frei von Kritik ist Erman allerdings auch gegenüber Maspero nicht. In seiner Biographie benennt er die Defizite, die er Maspero unterstellt und von denen er er-

ERMAN 1929, 289, 291, 272. Crum, Ransom Williams und Boeser werden nur als Studenten aus dem Ausland erwähnt, Francis LI. Griffith, Dévaud und Golenischeff tauchen in der Biographie Ermans gar nicht auf. Eugène Charles Revillout wird von ERMAN 1929, 204 und 260, in Zusammenhang mit einem Besuch bei ihm in Paris und seiner Anerkennung für Ermans Neuägyptischer Grammatik genannt, zu anderen Kollegen im Folgenden.

5 ERMAN 1929, 260. Zumindest teilweise wird man in diesem Zwist beiden Seiten auch persönliche Motive unterstellen können. Naville war bei der Wiederbesetzung der Stelle von Lepsius unterlegen (ebd., 170f.) und vertrat auch in dem Skarabäenstreit eine Meinung, die sich letztlich als nicht haltbar erwies, dazu im Folgenden.

6 Davon zeugt ein Briefwechsel zwischen Erman und Naville im April und Juni 1897.

7 ERMAN 1929, 215. 
klärt, dass Maspero sie auch an seine Schüler weitergegeben hätte. Vor allem zu schnelles Arbeiten und zu wenig Forschergeist zählt er dazu. ${ }^{8}$

Der Belgier Jean Capart findet in der Biographie Ermans wegen einer recht unglücklichen Fälschungsgeschichte Erwähnung, die trotz der konträren Standpunkte der beiden aber zu keiner Unterbrechung der Korrespondenz führte (s.a. noch im Folgenden).

Neben der zwar nicht sehr häufigen, aber umso dezidierteren Kritik insbesondere an Vertretern einer „französischen Ägyptologie“ fällt die große Herzlichkeit und Anerkennung auf, mit der Erman den englischen Ägyptologen Norman de Garis Davies würdigt. Ihn lernte Erman bei seinem zweiten Ägyptenbesuch 1899 kennen. Dazu schreibt er: ${ }^{9}$

„Eines kurzen Besuches in Sakkarah, wo ich in den großen Gräbern zu arbeiten hatte, gedenke ich aber gern. Denn da hauste im Grabe des Ptahhotep, unter einem Blocke, der jeden Augenblick den Einsturz drohte, in denkbar primitivster Weise ein Engländer, dessen fröhliches, ungebundenes Wesen mir vom ersten Augenblick an gefiel. Er war ein Theologe gewesen, dem aber die nötige Rechtgläubigkeit gefehlt hatte; so hatte er in der Welt umherirren müssen, bis die Gesellschaft des Archaeological Survey of Egypt ihn mit dem Abzeichnen der Gräber betraute. Seither ist N. de G. Davies eine Berühmtheit geworden, der Mann, der die Gräber von Tell Amarna veröffentlicht hat und der jetzt die herrlichen Veröffentlichungen der Thebanischen Gräber herausgibt. Wenn wir ihn früher im Unterschiede von einem amerikanischen Millionär gleichen Namens, der auch in Ägypten wirkte, den , armen Davies' nannten, so heißt er jetzt der ,richtige Davies'. Damals haben wir zusammen in jenem Grabe gehaust, und uns über unsere beiderseitigen Landsleute amüsiert, die von Stangen oder Cook angeschleppt wurden; auch deutsche Studentenlieder haben wir gesungen und der vornehme Wesir des Königs Assa und sein Sohn Achethotep, die dort bestattet sind, werden sich über diese vergnügten Barbaren gewundert haben."

Der briefliche Kontakt zwischen Erman und Davies wurde erst drei Jahre später (1902) aufgenommen und bestand bis zum Todesjahr Ermans. Die Mitteilungen beschränken sich dabei weitestgehend auf Berichte über die bevorstehenden oder abgeschlossenen Tätigkeiten und Dankessagungen für übersandte Werke, reflektieren also in keiner Weise den freundschaftlichen Umgang, den beide während ihres Aufenthaltes in Ägypten hatten (s. aber noch im Folgenden). 


\subsection{Die Inhalte der Korrespondenz}

In seinem ersten Brief vom 18. März 1889 aus München wandte sich Walter E. Crum an Adolf Erman, weil er bei ihm in Berlin Ägyptologie studieren wollte. ${ }^{10}$ Sein erklärtes und formuliertes Ziel war es zu der Zeit, eine Stelle am British Museum in London zu bekommen. Nach Berlin kam Crum nach einigen Verzögerungen dann tatsächlich und begründete damit einen Kontakt, der bis zum Tod Ermans bestand. Sein letzter (erhaltener) Brief datiert vom 18. Mai 1937 und ist an Frau Erman gerichtet. Er enthält Wünsche für den schwer Erkrankten, der einen Monat später am 26. Juni 1937 verstarb - Crum selbst starb am 18. Mai 1944. Briefe von Crum an Erman fehlen zwischen September 1914 und Juli 1916, aus dem Jahr 1918 sowie dem Jahr 1919 bis zum Sommer, als die Postverbindung zwischen Großbritannien und Deutschland unterbrochen war. Die Korrespondenz nach dem 1. Weltkrieg setzt mit einem Brief vom 11. August 1919 wieder ein. ${ }^{11}$

Am Beispiel der Briefe des englischen Koptologen Crum, der umfangreichsten Korrespondenz überhaupt, die an Erman gerichtet wurde, lässt sich nachzeichnen, worüber geschrieben wurde. Die bei Crum feststellbaren Themen finden sich so oder ähnlich auch in den Briefen anderer Kollegen, das Spektrum ist also vergleichbar. Verschiebungen ergeben sich durch die Gewichtung der Inhalte, zudem natürlich durch die Art und Hintergründe des jeweiligen Kontaktes und auch der jeweiligen Lebensumstände.

Breiten Raum nehmen in den Briefen von Crum wie auch in den anderen umfangreicheren Korrespondenzen persönliche und familiäre Angelegenheiten ein. Bei Crum wie auch in den Briefen anderer Kollegen kommen Familienangelegenheiten diverser Art zur Sprache: Krankheiten oder der Tod von Angehörigen werden mitgeteilt bzw. angesprochen, Verlobungen und Heiraten angezeigt. Die Lebensverhältnisse sind bekannt und/oder werden bekannt gemacht. Dazu gehört auch, dass der Lebensweg und Werdegang der Kinder erfragt und kommentiert wird. Crum wurde zudem Patenonkel von Peter, dem ältesten Sohn der Ermans, Alan H. Gardiner Patenonkel von Annemarie. Peter wird später im 1. Weltkrieg am 1. Juli 1916 an der Somme fallen, und Crum,

10 Der Kontakt von Crum und Erman könnte über Paris zustande gekommen sein. Crum hatte bei William N. Groff, einem amerikanischen Ägyptologen (1857-1901), in Paris studiert, dieser wiederum war 1878 Student bei Gaston Maspero in Paris gewesen, vgl. hierzu BIERBRIER 1995, 110 und 181.

11 Einen brieflichen Kontakt ohne größere Unterbrechungen hatte Erman während des 1. Weltkrieges, betrachtet man die umfangreicheren Korrespondenzen (s. Abb.), nur noch mit dem Schweizer Victor Dévaud. 
der an demselben Tag einen Neffen verlor, wird immer wieder anlässlich dieses beiderseitig erlittenen Verlustes zum 1. Juli an Erman schreiben. ${ }^{12}$

Gegenüber Erman spricht Crum auch das Scheitern seiner Ehe an - er verlässt 1910 seine Frau und England und geht mit seiner Lebensgefährtin nach Wien. In einem Brief vom 10. Dezember 1910 unterrichtet Crum schon von Wien aus Erman über diese Entwicklung, ganz offensichtlich in Erwartung einer Reaktion, die er nicht vorauszusehen vermag. ${ }^{13}$ Crums Lebensgefährtin, Margaret H(art) Davis bzw. Madge Hart-Davis ${ }^{14}$, lernte später Koptisch und wirkte ganz entscheidend bei Entstehung und Fertigstellung des Koptischen Wörterbuchs mit, das Crum von 1929 bis 1939 herausbrachte. Crum selbst tut sich schwer, sie in das Wissenschaftsgeschehen namentlich einzubringen. Im Vorwort zu seinem Koptischen Wörterbuch spricht er ihr zwar seinen tiefsten Dank aus (zumindest darf man vermuten, dass sie gemeint ist), nennt aber nicht ihren Namen. ${ }^{15}$

Dieser Austausch über persönliche und familiäre Ereignisse mag damit zusammenhängen, dass die Kontakte zwischen den Familien insgesamt recht eng waren. Erman empfängt in seinem Haus immer wieder Kollegen aus dem Ausland (auch Crum), Studenten von ihm halten sich dort sowieso häufig auf. ${ }^{16}$ Wolja Erichsen soll auf Vorschlag von Hans O. Lange und Kurt Sethe, die ihm in dieser Sache im Oktober 1932 schreiben, eine Zeitlang bei Erman wohnen allerdings nicht zuletzt, um der Beschlagnahme von Wohnraum zu entgehen. Vermutlich muss man in diesen Dingen aber auch einen anderen Stil unterstellen, eine grundsätzlich engere Verknüpfung von Beruflichem und Privatem und eine geringere Scheu, Privates in einen Brief einfließen zu lassen. So berichtet Gaston Maspero immer wieder einmal von seinem Sohn Henri, der Sinologe wurde und den Erman mit einiger Wahrscheinlichkeit gar nicht kennen gelernt hat oder wenn, dann als Kind.

12 Eine politische Dimension bekommt der Tod des Sohnes für Erman spätestens durch die Geschehnisse zu Beginn der 30er Jahre, vgl. dazu den Brief Ermans an den Rektor der Berliner Universität vom 30. Mai 1934, abgedruckt bei KLOFT et al. 1982, 43, Anlage XIII.

13 Die Antwort Ermans auf diese Mitteilung fällt offensichtlich gelassen, wenn nicht sogar wohlwollend aus. Jedenfalls bedankt sich Crum in einem Brief vom 15. Dezember 1910 für Ermans Reaktion und Freundschaft.

14 BIERBRIER 1995, 111 notiert ihren Namen als Madge Hart-Davis (1876-1953). Sie selbst unterschreibt in Briefen an Erman mit Margaret.

15 CRUM 1939, VIII. Die Unsicherheit Crums schlägt sich auch in einem Brief vom 11. März 1914 nieder, in dem er bei Erman anfragt, ob und wie in einer Besprechung von seiner Mitarbeiterin zu reden sei. Das Problem bestand für Crum vermutlich darin, dass er seine Freundin nicht heiraten konnte. Kollegen sprechen in ihren Briefen an Erman von ihr ganz selbstverständlich als Frau Crum. 
Das wissenschaftliche Tätigkeitsfeld Ermans und Ägyptologie als seine wissenschaftliche Disziplin kommen in der Korrespondenz auf verschiedene Weise zur Sprache.

Was sich in den Briefen von Crum ebenso wie von anderen Kollegen immer wieder findet, sind Anmerkungen zum Wissenschaftsbetrieb. Es wird sich über Personalia ausgetauscht, und es werden Diskussionen um Stellenbesetzungen geführt. Über das Netz, in das Erman mit seiner Korrespondenz eingebunden ist, erhält er so immer die neuesten Informationen über Entwicklungen im Fach. Zugleich unternimmt er über diese Kanäle auch diverse Versuche, auf übergeordneter Ebene eigene Interessen zu vertreten und z.B. Studenten oder Mitarbeiter an andere Stellen zu vermitteln. ${ }^{17}$

Auch die Zusammenarbeit an gemeinsamen Projekten schlägt sich in der Korrespondenz Ermans mit seinen internationalen Kollegen nieder, wobei damit vor allem anderen das Wörterbuch gemeint ist. ${ }^{18}$ Diese (formal) gemeinsam mit den Akademien Göttingen, Leipzig und München gestartete Unternehmung wurde seit ihrem Beginn von Erman dominiert, auf dessen Initiative sie ja auch zurückgeht. Bei Erman laufen die Fäden zusammen, und dies schlägt sich in den Briefen seiner Kollegen, und auch seiner Kollegen im Ausland sehr deutlich nieder.

Die internationale Herkunft seiner Studenten erwies sich für Erman als ausgesprochen hilfreich und ergiebig für die Arbeiten am Wörterbuch. Schon zu Studienzeiten, dann aber auch während ihrer weiteren wissenschaftlichen Zukunft und Karriere im Fach standen sie Erman in verschiedener Weise zur Verfügung, für Kollationierungen oder gänzlich neue Abschriften von Texten vor allem in der ersten Zeit. ${ }^{19}$ In den Briefen an sie wie auch an Kollegen, die mit der Zeit für das Projekt gewonnen werden konnten, geht es vor allem um die Absprache zu diesen Arbeiten, also eher um formale Vorgänge, in einem wesentlich geringeren Maße auch um inhaltliche Klärungen.

Späterhin wurde gerade James H. Breasted - zusätzlich zu seinen sonstigen Kompetenzen - in finanzieller Hinsicht zu einem wichtigen Partner, da er als Geldgeber für das Wörterbuch auftrat bzw. als Bindeglied zu Geldgebern fungierte. Auch Caroline Louise Ransom Williams wurde für Erman in dieser Weise - und auf ihre Bitte hin zumeist ohne Nennung ihres Namens - tätig.

17 Der Kontakt zu Maspero hat (u.a.) dazu beigetragen, dass Günther Roeder 1907 nach Ägypten ging.

18 ERMAN \& GRAPOW $1926 \mathrm{ff}$.

19 Gardiner etwa hielt sich längere Zeit u.a. in Leiden und Turin auf und füllte dort seine note-books. Das abgeschriebene Material ging dann an Erman in Berlin zur weiteren Bearbeitung für das Wörterbuch. 
Sie überwies immer wieder einmal Geld nach Berlin oder arrangierte andere, für das Wörterbuch günstige Regelungen und Unterstützungen. Entsprechend nimmt dieser Bereich einen größeren Teil in den Briefen von Breasted und Ransom Williams ein ${ }^{20}$, dann auch in denen von Lange.

Die Arbeiten am Wörterbuch und die infolgedessen immer weiter anwachsende Material- und Textsammlung in Berlin brachte es mit sich, dass Kollegen Anfragen an Erman richteten. Auch diese Anfragen waren oder wurden häufig zu einem Austausch auf Gegenseitigkeit.

Über gemeinsame Publikationsvorhaben war Erman z.B. mit Lange in Kopenhagen im Gespräch. In Zusammenhang mit seinen Arbeiten zu Asyūt und den Papyri aus al-Lahun stand Francis Ll. Griffith in einem regen Austausch mit Erman. Ein gemeinsames Vorhaben von Erman und Vladimir Golenischeff hingegen kam nicht zustande. Golenischeff war aus finanziellen Gründen gezwungen, seine Sammlung aufzugeben, und hatte ihren Verkauf nach Berlin angestrebt. Um das Bemühen und Scheitern dieses Transfers legen Briefe Golenischeffs aus den Jahren 1908 und 1909 Zeugnis ab.

Wie ein roter Faden ziehen sich durch die Briefe von Crum Berichte über laufende Arbeiten. Breiten Raum nimmt bei ihm die Arbeit am Koptischen Wörterbuch, seinem Lebenswerk, ein. Vom ersten Plan zu diesem Vorhaben über die Absprache mit seinem Kollegen Georg Steindorff bis hin zur Organisation und dem allmählichen Fortschreiten findet sich das Werden dieses Opus in den Briefen dokumentiert. Auch das Entstehen anderer Publikationen von Crum findet sich in den Briefen dokumentiert.

In ihren Briefen lassen auch andere Ägyptologen Erman an dem Werden und der Fertigstellung ihrer Arbeiten und Projekte teilhaben, mitunter auch an denen von Kollegen. Die jeweilige Sparte ist dabei nebensächlich. So berichtet z.B. Maspero von der Auffindung der Pyramidentexte in Pyramiden von Saqqāra im Winter 1880/81 wie auch von den Vorbereitungen zum Catalogue General, mit dem die Bestände des Kairener Museums veröffentlicht werden sollten, Édouard Naville und Norman de Garis Davies über ihre archäologischen Arbeiten, Griffith in einem Brief vom 4. Januar 1892 von der (wissenschaftlichen) Entdeckung H̦atnūbs durch Percy Newberry. In den Briefen von Gardiner werden die Bemühungen und das Zustandekommen des Sargtexteprojektes angesprochen, an dem neben ihm selbst Breasted und Pierre Lacau

20 Breasted hatte inzwischen den ersten Lehrstuhl für Ägyptologie in den USA inne, er wurde in Chicago eingerichtet. Ransom Williams, zeitweilig Studentin auch bei Breasted, arbeitete später am Museum in Toledo, lehrte zudem Ägyptologie und war an verschiedenen archäologischen Missionen in Ägypten beteiligt. 
beteiligt waren, und es lässt sich in ihnen die Entstehung der Egyptian Grammar beobachten.

Auch Erman hält seine Kollegen über sein Schaffen auf dem Laufenden. Ermans Artikel, Akademieschriften und Bücher, zudem Lieferungen des Wörterbuchs gingen an zahlreiche Kollegen, und Erman war in dieser Hinsicht offensichtlich sehr großzügig, wie die vielen Dankesbriefe zu erhaltenen Werken belegen. Häufig genug waren die erhaltenen Werke Anlass für die Kollegen, wieder einmal an Erman zu schreiben.

Zumindest in den Briefen von Kollegen aus dem Ausland spielen ein längerer wissenschaftlicher Austausch oder eine fortgesetzte wissenschaftliche Diskussion nicht die Rolle, die man vielleicht erwarten würde. Es werden selbstverständlich immer wieder fachliche Dinge besprochen, einzelne Wörter, Textstellen oder auch Transkriptionssysteme diskutiert, es wird sich auch schon einmal über das Werk eines Kollegen ausgetauscht oder eben die Organisation von Arbeit besprochen. Crum etwa diskutiert mit Erman sein Vorhaben eines Koptischen Wörterbuchs. Griffith, einer der führenden englischen Philologen seiner Zeit, äußert sich zum Verhältnis des Ägyptischen zu semitischen Sprachen oder auch zum ägyptischen Alphabet. Mit Lange tauschte sich Erman ebenso über Fragen der Lexikographie aus wie er mit ihm die Aufstellung eines Abgusses im Museum diskutierte, abgesehen davon, dass in Langes Briefen auch die gemeinsamen Publikationsprojekte Gegenstand der Diskussion waren. Dies alles geschieht insgesamt aber doch recht zurückgenommen. Über die Auffindung des Grabes von Tutanchamun entspinnt sich gar keine Diskussion, schon allein die Wahrnehmung dieses Fundes scheint recht eingeschränkt. Crum allerdings lässt Erman immer wieder Artikel britischer Zeitungen über den Fortgang der Arbeiten zukommen.

Mit Jean Capart hatte Erman das Pech, in eine wissenschaftliche Auseinandersetzung zu geraten, die dann auch in einigen Briefen angesprochen wird. ${ }^{21}$ Die Auseinandersetzung entzündete sich zwar nicht an den beiden Wissenschaftlern, d.h. sie gerieten nicht persönlich aneinander, die beiden gehörten aber - jedenfalls anfänglich - zu den führenden Kontrahenten in dem Streit. Gegenstand waren zwei Skarabäen, die von einer Umsegelung Afrikas zur Zeit Nechos berichten. Die Unterlagen im Nachlass Ermans umfassen dazu auch Photos und Abklatsche der Skarabäen, die sich über mehrere Korrespondenzen verstreut finden.

21 Die Kontroverse ist (u.a.) auch Gegenstand einiger Briefe von Alexandre Moret und Naville. 
Die Umstände, die zu dem Streit führen, legt Erman in seiner Biographie dar, wo sie im Detail nachzulesen sind. Dort heißt es u.a.:22

„Wieder vergingen Jahre, da kam im Frühjahr 1908 mein Kollege Heinrich Schäfer eines Tages zu mir und brachte mir einen Abklatsch einer Inschrift, die jener Herr [gemeint ist der Sohn von Urbain Bouriant] uns anbot. Sie stand auf einem Skarabäus von fabelhafter Größe und berichtete sehr Merkwürdiges, denn sie erzählte, daß die Flotte des Königs Necho Afrika umschifft habe - ganz so, wie das bei Herodot zu lesen steht. Daß eine Fälschung vorlag, hatte Schäfer schon gesehen, und als wir die Inschrift nun näher ansahen, zeigte es sich auch, daß sie von groben sprachlichen Fehlern wimmelte. Der Fälscher mußte ein Mann sein, der nur eine oberflächliche Kenntnis des Ägyptischen hatte. Was in seinem Fabrikate sprachlich richtig war, das hatte er aus echten Inschriften abgeschrieben; wo er auf eigene Faust Ägyptisch schrieb, wurde es fürchterlich. Mit Hilfe unseres Wörterbuches konnten wir leicht die sieben Quellen nachweisen, aus denen der Fälscher abgeschrieben hatte; ..."

Die Geschichte um die beiden gefälschten Skarabäen stieß in den Kernbereich der Ermanschen Arbeit vor. Sie betraf die Auseinandersetzung und Auffassungen um die ägyptische Sprache und den wissenschaftlichen Zugang zur ägyptischen Kultur überhaupt. An dieser Stelle offenbarte sich dann auch sehr deutlich der Zwist und die Kluft zu einigen ausländischen Kollegen, was Erman in seiner Biographie nicht verhehlt: ${ }^{23}$

„Was wir so über die Fälschung ermittelt hatten [Schäfer und Erman], legte ich der Akademie vor, und der Zufall wollte es, daß unser Aufsatz in den Tagen erschien, wo der internationale Historikertag in Berlin tagte. Auf dem aber hatte gerade ein enthusiastischer Fachgenosse diese neue große Entdeckung der Welt verkünden wollen. Davor konnte ich ihn noch bewahren; leider aber hatte die freche Fälschung schon vorher großes Unheil angerichtet. Denn Herr Bouriant hatte den Skarabäus, den er in Berlin nicht losgeworden war, auch andern Ägyptologen angeboten; Maspero hatte ihn glatt zurückgewiesen, bei anderen aber hatte er Glauben gefunden. Vor allem bei unserm Brüsseler Kollegen, Herrn Capart, der die beiden Skarabäen - denn das Monstrum war gleich in zwei Exemplaren auf die Welt gekommen - um 20000 Francs für sein Museum erworben hatte. Daß Herr Flinders Petrie in London an ihnen nicht zweifelte, konnte bei dessen Kritiklosigkeit und bei seiner Unkenntnis der Sprache nicht wundernehmen. Aber den wahren Triumph feierte der Fälscher erst, als Herr Capart die Skarabäen in Genf auf dem internationalen Geographentag vorlegte. Auf diesem Kongresse präsidierte der Genfer Ägyptologe Eduard Naville, und als dieser hörte, man erkläre in Berlin

22 ERMAN 1929, 245. Vgl. dazu auch FALSCHE FARAONEN 1983, 8-9. Ich danke Dietrich Wildung für diesen Hinweis.

23 ERMAN 1929, $245 f$. 
die Inschriften für falsch, weil sie so viele sprachliche Ungeheuerlichkeiten enthielten, da brach er los: die Inschriften seien natürlich echt; die école de Berlin, wie er uns auch sonst zu nennen liebte, verstände gar nichts, und ihre Grammatik sei eitel Unsinn. Der Auftritt mußte skandalös gewesen sein, denn mein Kollege Hellmann, der ihm beigewohnt hatte, forderte mich auf, dagegen zu protestieren. Das war nun freilich nicht mehr nötig, denn inzwischen war unser Aufsatz in den Sitzungsberichten der Akademie erschienen und hatte gezeigt, aus welchen Inschriften der Fälscher sein Machwerk zusammengestellt hatte. Seitdem zog man es vor, die Sache zu vergessen; daß Herr Naville oder Herr Petrie ihre Äußerungen zurückgenommen hätten, habe ich freilich nie gehört."

Auch für diesen Konflikt muss betont werden, dass er nicht oder nicht vorrangig in der Korrespondenz zwischen Erman und Capart ausgetragen wurde. Wenngleich man darüber brieflich Argumente austauschte, fand der eigentliche Konflikt dort statt, wo er sich auch entzündet hatte, in der Öffentlichkeit. Mit Capart bestand weiterhin brieflicher Kontakt, der erst 1921 abbrach. Auch mit Naville und Petrie, mit letzterem aber nur sehr eingeschränkt, tauschte sich Erman nach diesen Vorfällen noch brieflich aus, ohne dass die Zwistigkeiten allerdings eine Rolle gespielt hätten. Die Affäre vertiefte aber ohne Zweifel die nationalen Gräben und insbesondere die Kluft, die zwischen der Wissenschaftsauffassung der Berliner Schule und der die „école de Berlin“ ablehnenden frankophonen Ägyptologie bestand.

\section{Die deutsche Ägyptologie auf internationaler Ebene}

Jede der international geführten Korrespondenzen weist gewisse Charakteristika und Schwerpunkte auf, d.h. sie waren für Adolf Erman aus unterschiedlichen Gründen von Bedeutung und wurden von ihm durchaus mit Blick auf bestimmte Interessen geführt bzw. aufrechterhalten. Insofern sind sie wichtiges Instrumentarium der von Erman auf internationaler Ebene verfolgten Wissenschaftspolitik gewesen. Erman nutzte seine Kontakte zu Kollegen aus dem Ausland in vielfältiger Weise, um die deutsche Ägyptologie bzw. die von ihm dominierte deutsche Ägyptologie in das internationale Wissenschaftsgeschehen zu integrieren. Diese Bestrebungen wie auch die Stellung der deutschen Ägyptologie innerhalb des Faches als international betriebener Wissenschaft waren über die Jahre Veränderungen unterworfen, da sie natürlich nicht vom politischen Zeitgeschehen und dessen schon allein faktischen Auswirkungen losgelöst betrachtet werden können. 
Für die wissenschaftsgeschichtliche Forschung und die Definierung der wissenschaftsgeschichtlichen Stellung eines Faches zu einem bestimmten Zeitpunkt sind historische Ereignisse nicht notwendigerweise auch inhaltliche Einschnitte. Dies trifft m. E. gerade auch für den 1. Weltkrieg zu: Der politische Bruch, den der 1. Weltkrieg in verschiedener Hinsicht mit sich brachte, spiegelt sich nicht durch einen Bruch im Fach, in der Wissenschaft wider. Kontinuität herrschte in der Forschung, wie auch die Institutionen aus der Zeit vor dem Krieg nach 1918 weiter bestanden.

Aber natürlich blieb der 1. Weltkrieg nicht ohne Folgen für die Ägyptologie. Nehmen wir allein die zeitweilige Loslösung der deutschen Ägyptologen von ihren internationalen Kontakten. Die Postverbindungen waren unterbrochen oder wurden überwacht, so dass kein Austausch oder zumindest kein kontinuierlicher Austausch möglich war. Eine Reihe von Briefen scheint auch verloren gegangen zu sein. Die Unterbrechung in der Korrespondenz mit Walter E. Crum über anderthalb Jahre war dabei noch vergleichsweise gering (s. auch zuvor mit Anm. 11). Für Erman war diese Abtrennung vom internationalen Geschehen von besonders einschneidender Bedeutung, da er sein Projekt Wörterbuch international angelegt hatte und die Abschriften von Texten und auch die Verzettelung über ein international aufgebautes Kontakt- und Korrespondenznetz liefen.

In dem eingangs zitierten Abschnitt aus Ermans Biographie, in dem er von seinen ausländischen Studenten spricht, betont er zugleich, dass der Kontakt zu ihnen ,auch die schwerste Prüfung in der Kriegs- und Nachkriegszeit überstanden" habe. Offensichtlich war es diesen Kollegen möglich, persönliche und politische Ebene zu trennen - wirklich unpolitisch oder unparteiisch konnte man in dieser Situation wohl kaum sein. So erhält Erman nicht nur von Crum ein Kondolenzschreiben zum Tod seines Sohnes, sondern auch andere Kollegen aus Nationen, die am Krieg beteiligt waren, schreiben ihm, mitunter erst Jahre später. Crum fasste dieses Verhalten in seinem Kondolenzschreiben vom 18. Juli 1916 mit den Worten zusammen: „Der Krieg mag Völker scheiden; auf alte Freundschaften darf er nicht wirken", Norman de Garis Davies spricht in Zusammenhang mit der politischen Situation in einem Brief vom 3. August 1919 von der Unterscheidung zwischen Deutschen und Freunden. Auch Alan H. Gardiner geht in seinem ersten Brief nach dem Krieg vom 2. September 1919 auf den für beide Seiten sicher nicht leichten Neubeginn ein. Seine Position - dezidiert und beeindruckend vorgetragen - umfasst den ausdrücklichen Verzicht, die Ereignisse der zurückliegenden Jahre ("these years of bloodshed and misery") zu kommentieren. An Erman und Kurt Sethe richtet er die Worte: "my mouth is and ought to be closed, but you must judge of my 
opinions from your pre-war experience of my nature and character." Er betont, dass die persönliche Freundschaft unangetastet sei und beruft sich auf seinen unbeirrbaren Glauben an die Internationalität von Wissenschaft, die von solchen Ereignissen unberührt bleiben sollte.

Diese Toleranz der ausländischen Kollegen einerseits und sicherlich die Akzeptanz der Person Ermans schon vor dem Krieg andererseits erleichterten eine Rückkehr zu ,normalen“ Verhältnissen offensichtlich erheblich. Der briefliche Austausch, gerade auch für die Belange des Wörterbuchs, kam sehr schnell wieder in Gang.

Einschneidende Konsequenzen hatten die Ereignisse des 1. Weltkrieges für die Arbeit direkt vor Ort in Ägypten. Die Situation der deutschen Archäologen in Ägypten gestaltete sich ungleich schwieriger als die derjenigen, die in Deutschland an Universitäten, Akademien oder Museen arbeiteten. Mehrere Briefe legen beredtes Zeugnis davon ab, wie intensiv sich Erman für die Wiederaufnahme der deutschen Grabungen in Amarna einsetzte - und auf wieviel Ablehnung er mit seiner Offensive in dieser Sache stieß. Nachdem Gardiner bereits am 13. August 1920 an Erman geschrieben hatte, dass an die Vergabe einer Grabungskonzession an die Deutsche Orientgesellschaft nicht zu denken $\mathrm{sei}^{24}{ }^{24}$ folgte am 2. September 1920 eine weitere harsche Reaktion Gardiners auf das Anliegen Ermans wegen Amarna, von ihm im Sinne Ludwig Borchardts vorgetragen. ${ }^{25}$ Einen Vorstoß, sich für Erman bei Pierre Lacau zu verwenden, dem Nachfolger Gaston Masperos in der Leitung des Service des Antiquités, hatte Gardiner schon ein Jahr zuvor, in seinem Brief vom September 1919 abgelehnt. Gardiner musste sich selbst mit Lacau arrangieren, und zwar wegen des Sargtexteprojektes, das er und James H. Breasted planten. Gardiner vermutete, dass notwendige Absprachen für dieses Vorhaben mit Lacau schwierig sein würden (was sie dann doch nicht in dem angenommenen Maße waren) und mochte die Anfrage Ermans dort auch nicht weiterverfolgen. Trotz der in diesem Fall berechtigten Argumentation Gardiners, sich nicht für Erman einsetzen zu können, lassen seine Zeilen auch durchscheinen, als wie unangebracht das Anliegen Ermans eigentlich empfunden werden musste. Die deutsche Seite begnügt sich jedoch weitgehend damit, einen gewissen „Anti-

24 Ähnliche Briefe gingen auch an andere ausländische Kollegen, s. z.B. das Schreiben Ermans an den britischen Kollegen Francis Ll. Griffith vom 6. August 1920, abgedruckt bei KLOFT et al. 1982, 40, Anlage XI.

25 Mäßigend in der Sache versucht Sethe auf Erman zu wirken. Insbesondere in einem Brief vom 13. Oktober 1920 spricht er sich für eine Klärung mit Gardiner aus und tritt für Pierre Lacau ein, indem er auf die Leiden der Franzosen aufmerksam macht. Er äuBert sich zudem dahingehend, dass Lacau nicht ,französischer, sondern aegyptischer Beamter" sei. 
germanismus" ${ }^{26}$ zumindest Lacau unterstellt, zu diskutieren. Nach einem Treffen mit Lacau in Paris stellt Gardiner am 25. September 1920 nochmals fest, dass es Deutschland auf Grund der politischen Situation für einige Jahre nicht möglich sein wird, in Ägypten zu arbeiten. Diese Einschätzung von ihm findet sich auch noch in einem weiteren Brief, den er ein knappes Jahr später (am 15. August 1921) an Erman schrieb.

So blieb es dabei, dass den deutschen Ägyptologen keine Grabungen in Ägypten gestattet waren. Dies änderte sich erst allmählich zu Beginn der 20er Jahre. Wie ein Brief Borchardts vom 8. November 1923 belegt, scheint die Kontaktaufnahme zumindest zu den Franzosen aber immer noch schwierig gewesen zu sein. Die (Wieder-)Eröffnung des Deutschen Hauses in Theben, wichtiger Stützpunkt für die archäologische Arbeit vor Ort, fand überhaupt erst am 1. April 1927 und damit fast ein Jahrzehnt nach Kriegsende statt.

Die allmähliche Wiedereingliederung der Deutschen in die archäologische Szene Ägyptens wird von zwei Faktoren begleitet, die für diese Entwicklung vermutlich nicht ganz unerheblich waren, sie im Gegenteil wahrscheinlich sogar vorantrieben. Zum einen ist zu beobachten, dass es zwischen Engländern und Franzosen, den beiden großen, die Grabungstätigkeiten in Ägypten auf den verschiedenen Ebenen dominierenden Parteien, zunehmend zu Querelen kam, insbesondere was Stellenbesetzungen im „Service“ angeht. Zum anderen taucht eine neue Kraft - Macht kann man sie zu diesem Zeitpunkt noch nicht nennen - in der Archäologie auf. Das wachsende Nationalgefühl der Ägypter schlägt sich auch in einem neuen Bewusstsein nieder, was die Organisation und Verwaltung archäologischer Arbeit anbelangt. „Egypt for the Egyptians“, zitiert aus einem Brief Borchardts vom 28. Mai 1923, ist schon seinerzeit der entsprechende Slogan, und ein Gesetzesentwurf, den Günther Roeder in einem Brief vom 12. September 1924 erwähnt, soll die Ausfuhr von Aegyptiaca, bislang gängige Praxis, verhindern. Es wird zwar noch eine Weile dauern, bis die ägyptischen Belange mit entsprechenden Maßnahmen und Stellenbesetzungen realisiert werden können, doch ist der Weg dorthin nach dem 1. Weltkrieg und vor allem seit den 20er Jahren bereitet. Für die ägyptische Ägyptologie sind die Ereignisse in Europa und die damit einhergehenden Verschiebungen im politischen Machtgefüge also durchaus als Chance zu begreifen, zumal die Vorbehalte gegenüber einer europäischen Vereinnahmung des Landes bereits lange schwelten. Erman schreibt in seiner Biographie über das Ansehen

26 So Sethe in seinem schon in Anm. 25 erwähnten Brief an Erman vom 13. Oktober 1920. Auch Crum gibt schon am 21. Juni 1919 eine solche Einschätzung Heinrich Schäfers an Erman weiter. 
der Europäer in Ägypten schon 1885 anlässlich seiner ersten Ägyptenreise u.a.: ${ }^{27}$

„Auch die politischen Verhältnisse waren nicht erquicklich. In der arabischen Bevölkerung gärte es im geheimen, und wer nicht bloß in Hotels lebte oder mit dem Dragoman reiste, konnte wohl bemerken, welchen Haß die Leute gegen die Europäer hegten. Dabei machten sie aber doch einen Unterschied; am verhaßtesten waren die Inglisi, die Engländer; dann kamen die Franzosen; die Italiener und Griechen standen dem Volke näher, und von uns Brussiani, d.h. Preußen, hatten sie die unklare Vorstellung, daß wir den andern Feind und daher ihnen Freund sein müßten."

Auch wenn er in seiner Biographie von Ägyptern spricht, die „zuweilen“ zum Studium zu ihm nach Berlin kamen (s. zuvor), fand eine Korrespondenz Ermans mit ägyptischen Kollegen nahezu nicht statt. ${ }^{28}$ Dieser Umstand ist natürlich auch - aber sicher nicht nur - eine Altersfrage. Schon Selim Hassan (1886-1961), der als erster Ägypter eine Professur für Ägyptologie an der Universität Kairo bekam (1931) und später die Leitung des ägyptischen Antikendienstes übernahm, gehörte der nächsten Generation von Ägyptologen an. Die Ägypter, die nach ihm zur Ägyptologie kamen und von denen einige auch in Deutschland studierten, waren entsprechend noch jünger.

Die Zeit des aufkommenden Nationalsozialismus sei kurz angesprochen. Die von Adolf Hitler betriebene Politik einer Verfolgung von Randgruppen, insbesondere von Bürgern jüdischen Glaubens, führte innerhalb der deutschen Ägyptologie zwangsläufig zu Polarisierungen. Bis zum Tod von Erman im Juni 1937 blieben die Ereignisse in Deutschland auf die Korrespondenz mit seinen ausländischen Kollegen allerdings ohne größeren Einfluss.

Die Situation zu Ende der 20er und Beginn der 30er Jahre wird von außen schon aufmerksam beobachtet. Es finden sich z.B. in den Briefen Crums vermehrt Bemerkungen über die politische Situation und das politische Zeitgeschehen. Als „Ihr Hitler-Brief" wird von Crum am 4. Februar 1933 ein Brief Ermans zitiert. Offenbar hielt sich Erman mit Kommentaren und Bemerkungen nicht zurück, was ihm angesichts der erfahrenen Repressalien auch schwer gefallen sein dürfte. Auch wird die Emigration deutscher Kollegen, wie ein Brief Hans O. Langes vom Mai 1934 belegt, zur Kenntnis genommen. Aus den Briefen Langes kommt großes Mitgefühl für die Lage seines Berliner Kollegen und Freundes zum Ausdruck, als dessen Tätigkeitsbereich mehr und mehr eingeschränkt wird. Insgesamt aber lassen die Briefe aus dem Ausland vor

27 ERMAN 1929, 206.

28 Ahmed Badawi (1 Brief), Sami Gabra (beschriebene Visitenkarte), Selim Hassan (2 Briefe) und Ahmed Kamal (1 Brief) wandten sich an Erman. 
allem eine gewisse Hilflosigkeit erkennen und den diffusen (und letztlich nicht begründeten) Glauben, dass alles schon wieder besser werden würde. Unklar bleibt auch, ob und welche Erwartungen Erman selbst in dieser Sache an seine Kontakte mit ausländischen Kollegen knüpfte.

\section{Schlusswort}

Der Nachlass Adolf Ermans in der SuUB Bremen gibt wichtige, die Hinweise in seiner Biographie ergänzende Informationen dazu, mit welchen Kollegen Erman auf internationaler Ebene korrespondierte und worüber. Dies gilt unabhängig davon, dass neben Fragen zum erhaltenen Umfang der Korrespondenz (s. einleitend) sich auch an die Inhalte der Briefe gewisse Unsicherheiten knüpfen. Nicht in jedem Fall wird aus den Worten des Korrespondenzpartners wirklich ersichtlich, worum es im Detail geht. Und es ist nicht unbedingt erkennbar, was den Anteil Ermans an dem einzelnen Vorgang ausmachte und worin dessen Interesse an dem jeweiligen Briefwechsel lag. Gerade die sehr ausführliche Korrespondenz mit Francis Ll. Griffith lässt in dieser Hinsicht manches offen. Um hier weitere Erkenntnisse zu erlangen, sind zusätzliche Recherchen auch außerhalb des Nachlasses notwendig. Hilfreich wäre es dabei möglicherweise auch, Einblicke in Gegenkorrespondenzen zu nehmen, sofern dies möglich ist.

Einfluss und Wirkung Ermans in der Ägyptologie waren - darüber bedarf es keiner Diskussion - enorm. Mit seinen Schülern, die aus dem Ausland zu ihm kamen (alle etwa um die Jahrhundertwende), begründete Erman auch international ein Netzwerk, das er pflegte und das für ihn, für seine Forschungen und vor allem für sein Vorhaben Wörterbuch von ausgesprochen großem Nutzen war. Dieses Netzwerk hat Erman weiter und offensiv ausgebaut. Die Korrespondenz mit seinen (ehemaligen) Schülern wie auch mit anderen Kollegen, die nach und nach dazukamen, zeigt zudem sehr deutlich, wie bei Erman die Fäden zusammenliefen. Dies betrifft den direkten Austausch, aber auch Kontaktaufnahmen zwischen Kollegen liefen des Öfteren über Erman.

Die Rezeption seiner Auffassung von Wissenschaft außerhalb Deutschlands war indes unterschiedlich. Mit seinem Streben, sich der kritischen, wissenschaftlichen Erforschung des alten Ägyptens zu widmen, entfernte er sich gewollt immer weiter von der bisherigen schwärmerischen Auseinandersetzung mit einer in ihrer offiziellen Selbstdarstellung prunkvollen und inszenier- 
ten Kultur. ${ }^{29}$ Diese Einstellung und insbesondere ihre Auswirkungen auf die altägyptische Sprachwissenschaft fand nicht überall Anhänger.

Das politische Zeitgeschehen verschärfte nationale Spannungen, wenngleich durch die persönlichen Kontakte, die Erman mit seinen ausländischen Kollegen schon lange vor dem 1. Weltkrieg pflegte, die Korrespondenz mit ihnen nach 1918 recht zügig wieder in Gang kam. Folgen hatten die Ereignisse vor allem auf die archäologische Arbeit in Ägypten selbst, der 1. Weltkrieg brachte hier grundlegende Einschnitte mit sich. Diese konnten zuvor nur in Grundzügen umrissen werden. Durch die Hinzunahme von Informationen, die aus den übrigen Korrespondenzen gewonnen werden können, und durch eine gezielte Verknüpfung historischer Ereignisse mit den brieflichen Darstellungen ließe sich die Stellung der deutschen Ägyptologie innerhalb des internationalen Geflechts noch weiter verfeinern.

\section{Literatur}

BIERBRIER 1995. Bierbrier, Moritz L. (Hg.), Who was who in Egyptology?, London ${ }^{3} 1995$.

CRUM 1939. Crum, Walter Ewing, A Coptic Dictionary, Oxford 1939.

ERMAN 1929. Erman, Adolf, Mein Werden und mein Wirken. Erinnerungen eines alten Berliner Gelehrten, Leipzig 1929.

ERMAN \& GRAPOW 1935-1953. Ders. \& Hermann Grapow, Wörterbuch der aegyptischen Sprache, 7 Bände, Berlin \& Leipzig 1926-1963, Belegstellen 5 Bände, Berlin \& Leipzig 1935-1953.

FALSCHE FARAONEN 1983. Falsche Faronen. Zeitung zur Sonderausstellung 400 Jahre Fälschungsgeschichte, Staatliche Sammlung Ägyptischer Kunst, München 21.7.31.10.1983.

GESTERMANN \& SChIPPER 2004. Gestermann, Louise \& Bernd U. Schipper, Der Nachlaß Adolf Ermans in der Staats- und Universitätsbibliothek Bremen, Göttinger Miszellen 201 (2004), 37-48.

KLOFT et al. 1982. Kloft, Hans (Hg.), Der Nachlass Adolf Erman (unter Mitarbeit von Thomas Elsman und Sabine Gorsemann), (Veröffentlichungen der Abteilung Gesellschaftswissenschaften und der Spezialabteilung 38), Bremen 1982.

RÜHLMANN 1988. Rühlmann, Gerhard, Gedanken zu Adolf Ermans Werk, Ägypten und ägyptisches Leben im Altertum', Zeitschrift für Ägyptische Sprache und Altertumskunde 115 (1988), 157-160. 